\title{
Conjugation of Hot-Melt Extrusion with High-Pressure Homogenization: a Novel Method of Continuously Preparing Nanocrystal Solid Dispersions
}

\author{
Xingyou Ye, ${ }^{1}$ Hemlata Patil, ${ }^{1}$ Xin Feng, ${ }^{1}$ Roshan V. Tiwari, ${ }^{1}$ Jiannan Lu, ${ }^{1,5}$ Andreas Gryczke, ${ }^{2}$ Karl Kolter, ${ }^{3}$ \\ Nigel Langley, ${ }^{4}$ Soumyajit Majumdar, ${ }^{1,6}$ Dipesh Neupane, ${ }^{7}$ Sanjay R. Mishra, ${ }^{7}$ and Michael A. Repka ${ }^{1,6,8}$
}

Received 27 May 2015; accepted 6 August 2015; published online 18 August 2015

\begin{abstract}
Over the past few decades, nanocrystal formulations have evolved as promising drug delivery systems owing to their ability to enhance the bioavailability and maintain the stability of poorly watersoluble drugs. However, conventional methods of preparing nanocrystal formulations, such as spray drying and freeze drying, have some drawbacks including high cost, time and energy inefficiency, traces of residual solvent, and difficulties in continuous operation. Therefore, new techniques for the production of nanocrystal formulations are necessary. The main objective of this study was to introduce a new technique for the production of nanocrystal solid dispersions (NCSDs) by combining high-pressure homogenization (HPH) and hot-melt extrusion (HME). Efavirenz (EFZ), a Biopharmaceutics Classification System class II drug, which is used for the treatment of human immunodeficiency virus (HIV) type I, was selected as the model drug for this study. A nanosuspension (NS) was first prepared by HPH using sodium lauryl sulfate (SLS) and Kollidon ${ }^{\circledR} 30$ as a stabilizer system. The NS was then mixed with Soluplus ${ }^{\circledR}$ in the extruder barrel, and the water was removed by evaporation. The decreased particle size and crystalline state of EFZ were confirmed by scanning electron microscopy, zeta particle size analysis, and differential scanning calorimetry. The increased dissolution rate was also determined. EFZ NCSD was found to be highly stable after storage for 6 months. In summary, the conjugation of HPH with HME technology was demonstrated to be a promising novel method for the production of NCSDs.
\end{abstract}

KEY WORDS: efavirenz; high-pressure homogenization; hot-melt extrusion; nanocrystal solid dispersion; nanosuspension.

\section{INTRODUCTION}

More than $40 \%$ of drugs developed by the pharmaceutical industry are poorly soluble in water $(1,2)$. With the introduction of high-throughput screening (HTS) methods for the

\footnotetext{
${ }^{1}$ Department of Pharmaceutics and Drug Delivery, School of Pharmacy, The University of Mississippi, University, Mississippi 38677, USA.

${ }^{2}$ BASF SE, Global Development and Technical Marketing, Ludwigshafen, 67056, Germany.

${ }^{3}$ BASF SE, R\&D Product Management Excipients, Ludwigshafen, 67056, Germany.

${ }^{4}$ BASF Corporation, 500 White Plains Road, Tarrytown, New York 10591, USA.

${ }^{5}$ Pharmaceutical Application Laboratory, Shin-Etsu Chemical, Co, Ltd., Totowa, New Jersey 07512, USA.

${ }^{6}$ Pii Center for Pharmaceutical Technology, The University of Mississippi, University, Mississippi 38677, USA.

${ }^{7}$ Department of Physics, University of Memphis, Memphis, Tennessee 38152, USA.

${ }^{8}$ To whom correspondence should be addressed. (e-mail: marepka@olemiss.edu)
}

discovery of new drugs (3), the amount of poorly watersoluble drugs is increasing rapidly. Most drugs are administrated orally, as it is the most common and convenient route for drug delivery, so poor solubility often leads to poor gastrointestinal absorption, and thus poor bioavailability $(4,5)$. Solubility is also a critical parameter to be considered in parenteral formulations (6). Thus, enhancement of the dissolution rate of poorly soluble drugs remains one of the most challenging tasks for pharmaceutical scientists.

Various approaches have been employed to improve the dissolution rate of certain poorly water-soluble drugs. For example, salt formation is a chemical method used to make a drug into a prodrug to improve the dissolution rate. However, this method can only be applied to weakly acidic or basic drugs, not those with neutral $\mathrm{pH}$ (5). Incorporating drug molecules into cyclodextrin is also an effective way to enhance their dissolution rate, but this method requires a certain molecular size and conformation (7). The currently available dissolution rate enhancement methods are limited to drugs with certain properties such as acid-base properties, molecular size, and conformation.

Production of a solid dispersion (SD) is one of the most extensively studied methods of improving the dissolution rate 
of poorly soluble drugs. A SD can be defined as a solid system of a poorly water-soluble drug or drugs dispersed in an inert carrier, with the drug usually in the amorphous state $(5,8)$. The amorphous form of drug usually has more free energy than the crystalline counterpart, which leads to an increased dissolution rate (9). However, this high free energy also tends to create thermodynamic instability, which is a major drawback of amorphous SDs. Recrystallization is usually observed during the storage of SDs (9-11).

In order to solve the recrystallization problem, initially formulating drugs into a crystalline state in a smaller batch is a feasible method. It has been reported that the micronization of drug powders to particle sizes of between 1 and $10 \mu \mathrm{m}$ is not sufficient to overcome bioavailability problems of very poorly soluble drugs (12), so the natural progression is to move from micronization to nanonization, the production of nanocrystals. Nanocrystals are crystals with a particle size below $1000 \mathrm{~nm}$. The reduction in particle size increases the surface area, which directly affects the dissolution rate. Thus, by decreasing the particle size, a higher dissolution rate can be achieved. This can be explained with the help of the Noyes-Whiney equation (13):

$\frac{d W}{d t}=\frac{D A\left(C_{\mathrm{s}}-C\right)}{L}$

where $d W / d t$ is the rate of dissolution, $A$ is the surface area of the solid, $C$ is the concentration of the solid in the bulk dissolution medium, $C_{\mathrm{s}}$ is the concentration of the solid in the diffusion layer surrounding the solid, $D$ is the diffusion coefficient, and $L$ is the diffusion layer thickness.

However, it has been reported that decreasing the particle size below a critical value of $1-2 \mu \mathrm{m}$ increases the saturation solubility (12). This can be derived from the Kelvin equation, below, which is usually used to describe the vapor pressure of a curved droplet but can also be used to describe the dissolution process, in which $P$ and $P_{0}$ represent the actual and saturated dissolution pressure (14):

$\ln \frac{P}{P_{0}}=\frac{2 \gamma V_{\mathrm{m}}}{r R T}$

where $\gamma$ is the surface tension, $V_{\mathrm{m}}$ is the molar volume of the liquid, $R$ is the universal gas constant, $r$ is the radius of the particle, and $T$ is the temperature. As it can be seen from the equation, $P_{0}$ directly depends on $r$. A decrease in $r$ means a decrease in the particle size, which will increase the saturated dissolution pressure and thus increase the saturated solubility.

The methods for production of nanocrystals can be divided into two basic types: bottom-up technologies (controlled precipitation/crystallization) and top-down technologies (media milling/high-pressure homogenization [HPH]) (15). Currently, five nanocrystal products have been approved by the US FDA, all of which are based on top-down technologies (four of these products are prepared by wet ball milling and one is prepared by high-pressure homogenization) (16), which indicates that the top-down process is more industrially feasible (17). After completion of these processes, the drugs are present in a suspended state as a nanosuspension (NS). However, it is usually important for the NS to be transformed into a solid product, both for physical stability and for patient convenience $(16,18)$. The most widely used processes for transformation include freeze-drying and spray drying (19). However, these two methods have several disadvantages, such as high cost, time and energy inefficiency, and residual solvent trace (20). In addition, it is difficult to make the production process continuous. There is therefore a need for a new technology to complete the transformation process.

Hot-melt extrusion (HME) is considered as a promising technique in the pharmaceutical industry because of its advantages compared to other conventional techniques, such as being a solvent-free, continuous process with no timeconsuming steps $(21,22)$. These characteristics make HME an appropriate candidate for the solidification of NSs. HME has been used to make a variety of dosage forms, including pellets (23), tablets (24), transdermal films $(25,26)$, implants (27), and solid lipid nanoparticles (SLNs) $(28,29)$. However, the preparation of nanocrystal solid dispersions (NCSDs) by combing HPH and HME is not yet explored much (30).

Hot-melt extrusion technology has been previously utilized by Baumgartner et al. 2014 to prepare a nanoformulation; however, the authors used a media milling technique to prepare the nanosuspension, which was further processed through the hot-melt extruder to convert it into nanocrystals. In this 2014 study, the media milling technique utilized to prepare the nanosuspension was a time-consuming process taking about $24 \mathrm{~h}(30)$. However, the primary objective of the current study was to develop a new, less time consuming method for preparing a NCSD by using HME technology after $\mathrm{HPH}$. The first step was to prepare a NS using HPH, and then the resulting suspension was extruded via HME with the help of a selected polymer (Soluplus ${ }^{\circledR}$ ) to obtain a NCSD. This overall process took less than $1 \mathrm{~h}$ to complete. In addition, various physical and chemical properties were evaluated within the NCSD. Finally, the stability of the NCSD extrudates was studied for the first time (30).

\section{MATERIALS}

Efavirenz (EFZ) was purchased from Ria International LLC (East Hanover, NJ, US). Sodium lauryl sulfate (SLS) was purchased from Fisher Scientific (Hanover Park, IL, US). Kollidon ${ }^{\circledR} 30$ and Soluplus ${ }^{\circledR}$ were kindly donated by BASF SE (Ludwigshafen, Germany). All other reagents used in this study were of analytical grade.

\section{METHODS}

\section{Preliminary Study}

A preliminary study was carried out to evaluate the formulation and process parameters to be used in the preparation of the NCSD. The three most critical parameters in the HPH process were found to be drug concentration, homogenization pressure, and time, while in the HME process, the parameters taken into consideration were the ratio of NS to polymer, feeding rate, barrel temperature, screw configuration, screw speed, and zone for NS addition. All of these parameters were optimized to successfully prepare a NCSD by HPH-HME technique. 
Table I. Formulation Compositions of NS and Extrudate

\begin{tabular}{|c|c|c|c|c|c|}
\hline \multicolumn{2}{|l|}{ Formulation } & $\begin{array}{l}\text { Soluplus }{ }^{\circledR} \\
\%(w / w)\end{array}$ & $\begin{array}{l}\text { Kollidon }{ }^{\circledR} 30 \\
\%(w / v) \text { for } \mathrm{NS}, \\
\%(w / w) \text { for extrudate }\end{array}$ & $\begin{array}{l}\text { SLS } \\
\%(w / v) \text { for NS, } \\
\%(w / w) \text { for extrudate }\end{array}$ & $\begin{array}{l}\text { EFZ } \\
\%(w / v) \text { for NS, } \\
\%(w / w) \text { for extrudate }\end{array}$ \\
\hline NS & A & - & 1.0 & 0.5 & 2.0 \\
\hline & $\mathrm{B}$ & - & 1.0 & 0.5 & 4.0 \\
\hline Extrudate & A & 98.25 & 0.5 & 0.25 & 1.0 \\
\hline & B & 97.25 & 0.5 & 0.25 & 2.0 \\
\hline
\end{tabular}

$E F Z$ efavirenz, $N S$ nanosuspension, $S L S$ sodium lauryl sulfate

\section{Preparation of EFZ NS}

EFZ NS was prepared by HPH technique. Briefly, different concentrations of EFZ powder ( 2 and $4 \%, w / v)$ were poured into an aqueous surfactant solution (Kollidon ${ }^{\circledR} 30$, $1.0 \% w / v$ and SLS $0.5 \% w / v)$ (31) and further mixed on a magnetic stirrer at $500 \mathrm{rpm}$ for $12 \mathrm{~h}$ in order to achieve complete dispersion of EFZ in the solution. After dispersion, the mixtures were homogenized using a T25 digital ULTRATURRAX® basic homogenizer (IKA-Werke, Staufen, Germany) at 15,000 rpm for $5 \mathrm{~min}$. The resulting suspension was further homogenized using an EmulsiFlex-05 high-pressure homogenizer (Avestin Inc., Ottawa, Canada) at 1500 bar for selected cycles.

\section{Preparation of EFZ NCSD}

NCSD was prepared by mixing aqueous phases of NS and polymer (Soluplus $\left.{ }^{\circledR}\right)$ using an 11-mm co-rotating twin-screw extruder (Thermo Fisher Scientific, Waltham, MA, USA). The extruder consists of eight zones, the individual temperature of which, excluding zone 1, can be precisely controlled. The barrel was maintained within a temperature range of 100$140^{\circ} \mathrm{C}$. Soluplus ${ }^{\circledR}$ was fed into the extruder via an $11-\mathrm{mm}$ single-screw feeder (Thermo Electron, Karlsruhe, Germany) at a feeding rate of $1 \%$ (approximately $1.2 \mathrm{~g} / \mathrm{min}$ ). NS was injected into zone 5 using a $520 \mathrm{~S}$ pump (Watson Marlow, Golden, Colorado, US) at $0.3 \mathrm{rpm}$ (approximately $0.6 \mathrm{~g} /$ $\mathrm{min})$. The screw speed was set at $50 \mathrm{rpm}$.

\section{CHARACTERIZATION AND EVALUATION}

\section{Solubility of EFZ}

The solubility of EFZ was assessed at room temperature. EFZ (20 mg) was added to glass vessels containing $20 \mathrm{~mL}$ of ultrapurified water. The glass vessels were then placed on an MTS 2/4 microtiter shaker (IKA, Wilmington, NC, US) operated at a speed of $200 \mathrm{~min}^{-1}$ for $24 \mathrm{~h}$. The samples were then withdrawn and centrifuged at $6000 \mathrm{rpm}$ for $5 \mathrm{~min}$. The supernatant was analyzed using a GENESYS 6 ultraviolet-visible (UV-vis) spectrophotometer (Thermo Scientific, Madison, WI, US). The experiment was then repeated at least three times for all the samples. The means and standard deviations were then calculated.

\section{Particle Size and Zeta Potential}

As stated above, particle size can significantly affect the dissolution velocity and saturation solubility. Zeta potential is one of the critical parameters affecting the stability of NS (32). These two parameters were measured using a Zetasizer Nano ZS (Malvern Instruments, Worcestershire, UK). The NS sample was first diluted with sufficient ultra-purified water, and then the diluted sample was transferred to a cuvette for measurement. Dynamic light scattering was used to measure the $\mathrm{Z}$ average and polydispersity index (PDI). Laser Doppler

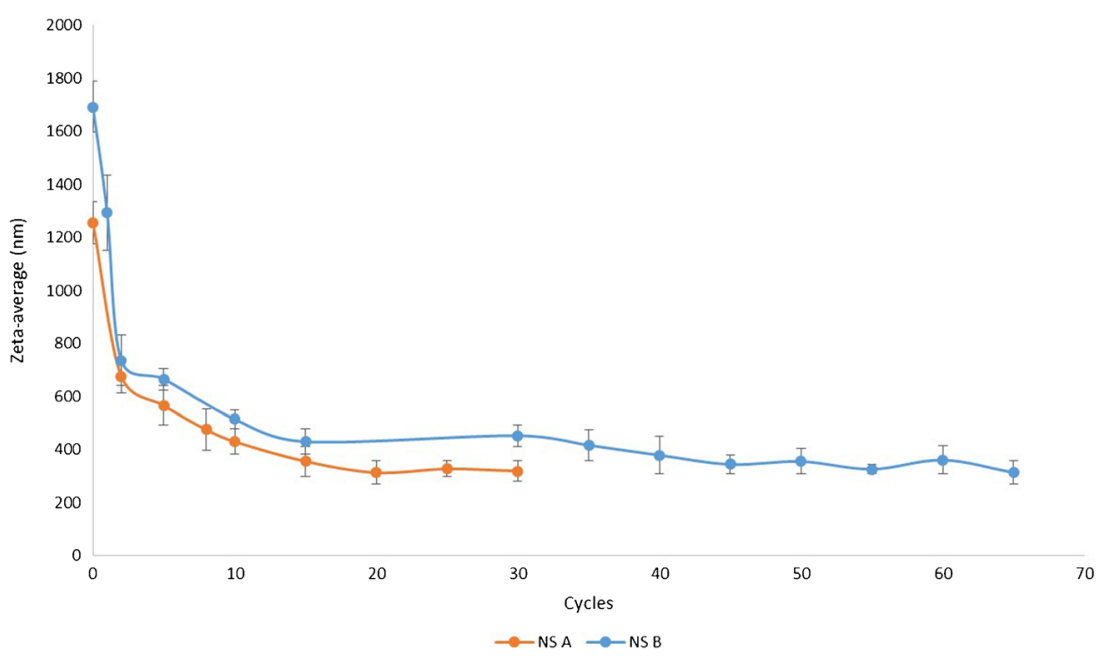

Fig. 1. Particle size versus cycle curves for nanosuspension $A$ and $B$ 


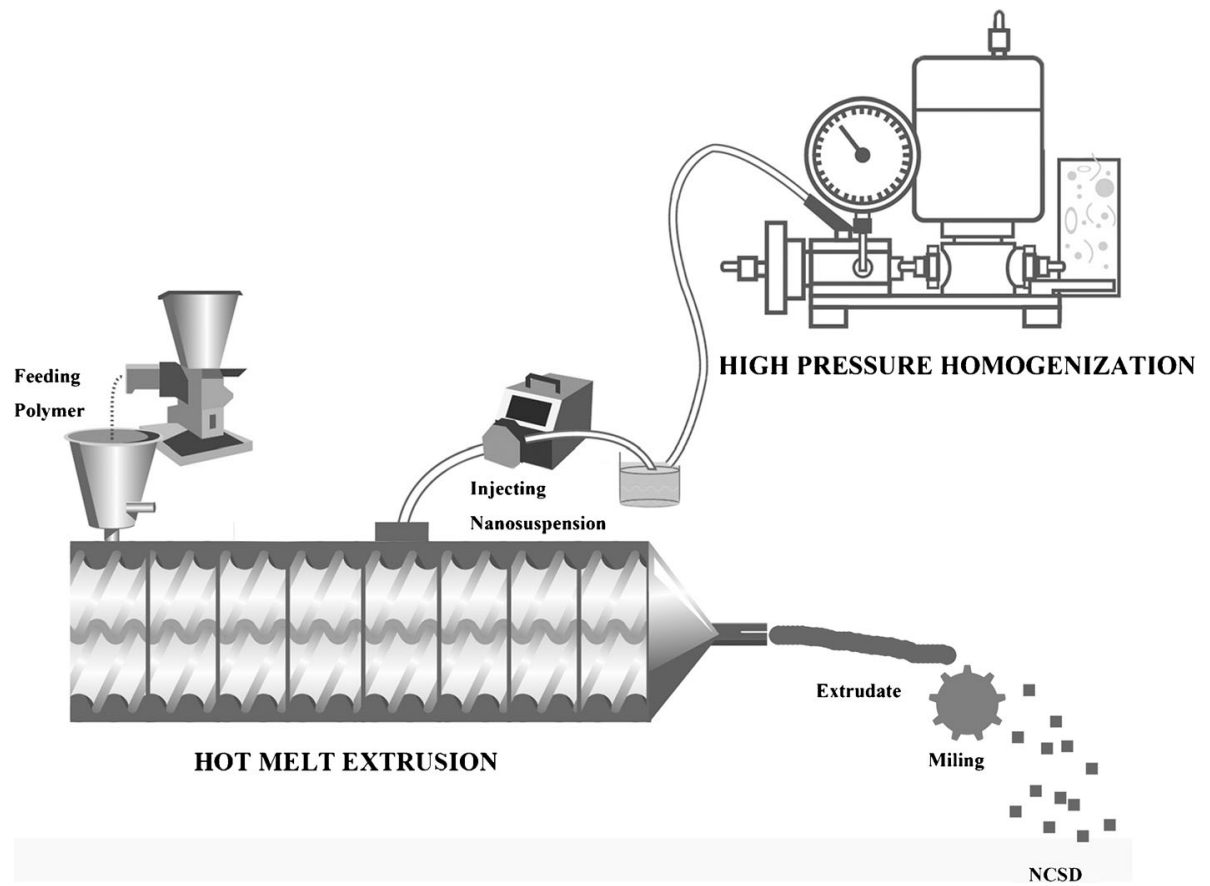

Fig. 2. Schematic representation of continuous preparation of a nanocrystal solid dispersion using a high-pressure homogenizer and hot-melt extruder

microelectrophoresis was used to measure the zeta potential. The parameters used for measurement were scattering angle of $173^{\circ}$, refractive index of 1.33 , viscosity of $0.89 \mathrm{cP}$, and temperature of $25^{\circ} \mathrm{C}$.

\section{Loss on Drying}

Soluplus ${ }^{\circledR}$ was extruded using identical processing conditions to those used for NCSD, and then NCSD and Soluplus ${ }^{\circledR}$ were

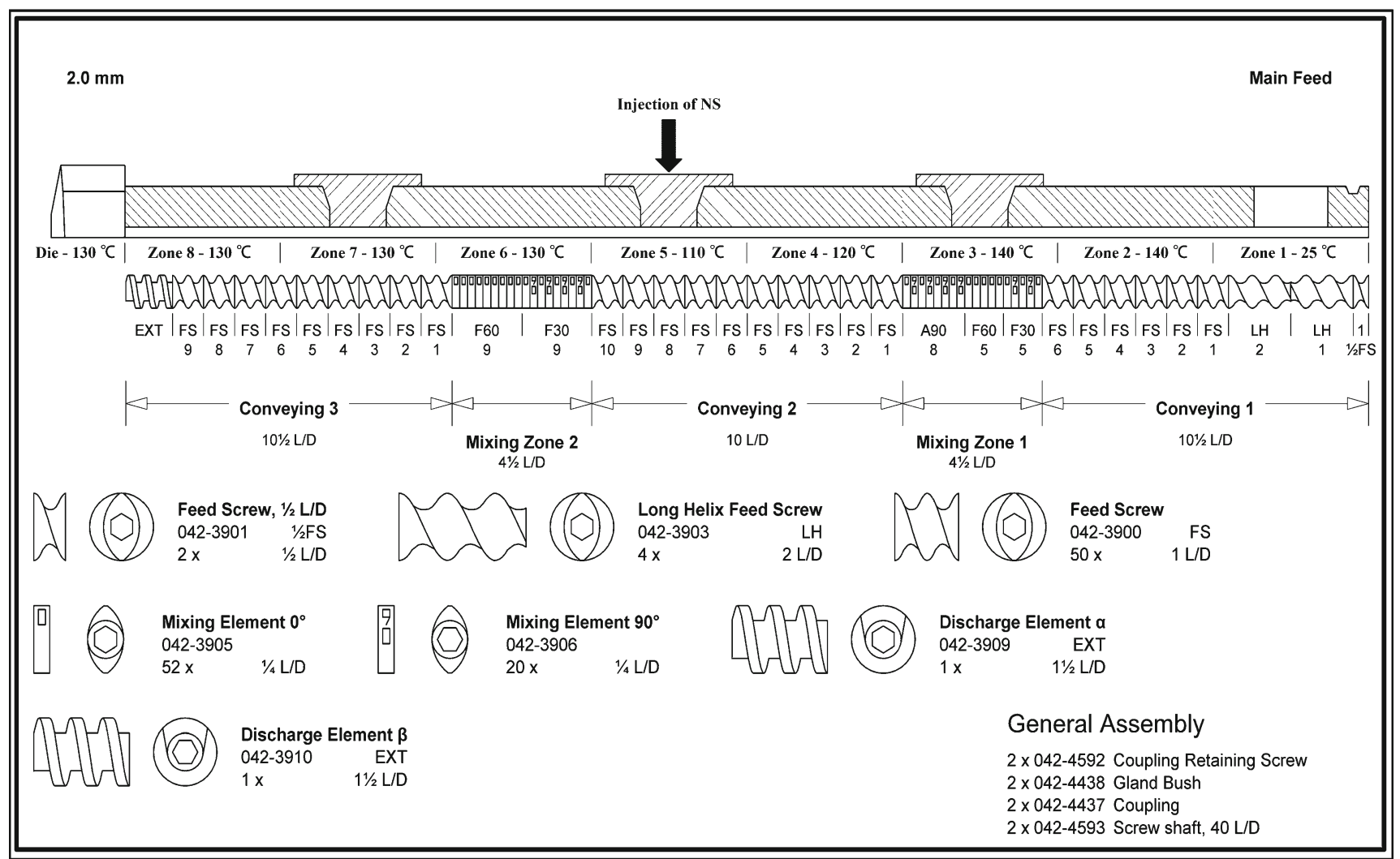

Fig. 3. Screw configuration used in the preparation of nanocrystal solid dispersions by hot-melt extrusion 

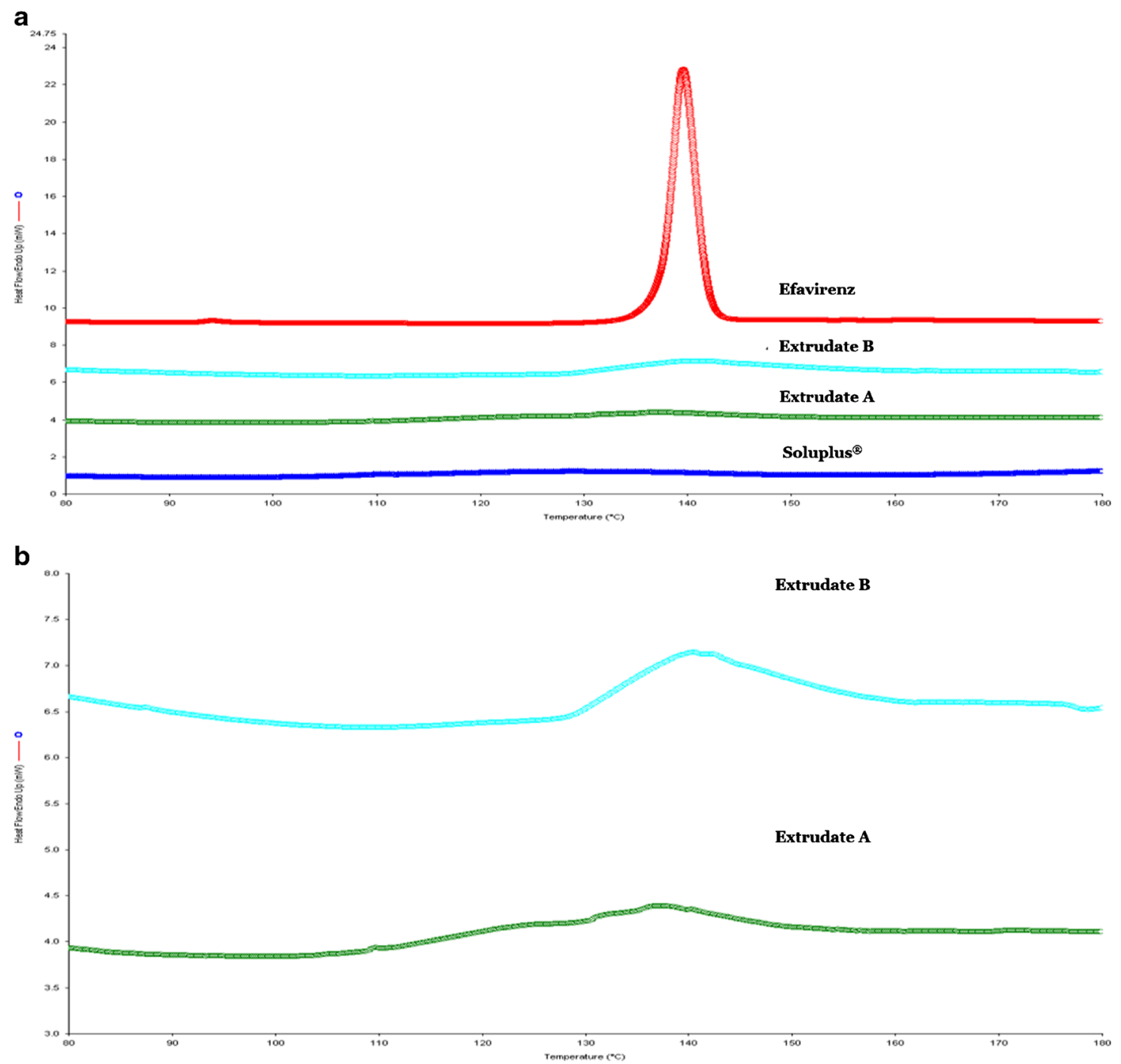

Fig. 4. Evaluation of crystallinity using differential scanning calorimetry. a Comparison of pure efavirenz, extrudate A (1.02\% drug loading), extrudate B (2.06\% drug loading), and Soluplus®. b Magnified differential scanning calorimetry thermograph for comparison of extrudates $\mathrm{A}$ and $\mathrm{B}$

subjected to a loss on drying (LOD) test using a MB45 Moisture Analyzer (Ohaus, Switzerland) by heating at $110^{\circ} \mathrm{C}$ for $10 \mathrm{~min}$.

\section{Differential Scanning Calorimetry}

A Diamond DSC (PerkinElmer, Shelton, CT, US) was used to measure the degree of crystallinity of the samples. The instrument's Pyris manager software was utilized to analyze the data. About 2-5 mg of samples were weighed and hermetically sealed in an aluminum pan. The heating rate was set at $20^{\circ} \mathrm{C} / \mathrm{min}$ from 20 to $200^{\circ} \mathrm{C}$ under an inert atmosphere of nitrogen at a flow rate of $20 \mathrm{~mL} / \mathrm{min}$.

\section{Drug Content}

The extrudate was first milled to a fine powder. Accurately weighed powder $(10 \mathrm{mg})$ was dissolved in $10 \mathrm{~mL}$ of methanol and then diluted 10 times with methanol. The sample was analyzed by UV-vis spectrophotometry.

\section{Scanning Electron Microscopy}

Scanning electron microscopy (SEM) was used to determine the morphology of pure EFZ and EFZ NCSD. Adhesive carbon tape was used to mount the sample onto an aluminum stage, and then the samples were sputter coated with gold under an argon atmosphere using a Hummer 6.2 Sputter Coater (Ladd Research Industries, Williston, VT, US). The coater was kept in a high-vacuum evaporator equipped with an omni-rotary stage tray to guarantee a uniform coating. Finally, images were captured using a JSM-5600 scanning electron microscope (JEOL USA, Inc., Waterford, VA, US) at an accelerating voltage of $5 \mathrm{kV}$.

\section{In Vitro Drug Release Study}

The dissolution media used was $900 \mathrm{~mL}$ of $0.2 \%$ SLS in 0.1 M HCL ( $\mathrm{pH}$ 1.2, simulated gastric medium) (33). SR8plus $^{\mathrm{TM}}$ dissolution apparatus (Hanson, Chatsworth, CA, US) 



Fig. 5. Scanning electron microscopy images of a pure efavirenz and $\mathbf{b}$ efavirenz nanocrystal solid dispersion (nanocrystals are marked by arrows)

was maintained at $37 \pm 0.5^{\circ} \mathrm{C}$ and the paddle speed was set at $50 \mathrm{rpm}$. Milled extrudates equivalent to $10 \mathrm{mg}$ of EFZ were filled into capsules (size 0) for dissolution. Samples were collected at intervals of 5, 15, 30, 60, and 120 min through a stainless steel cannula with a $0.2-\mu \mathrm{m}$ nylon filter tip attached to a $2.5-\mathrm{mL}$ syringe. The samples were analyzed directly using a UV-vis spectrophotometer.

\section{UV Analysis}

Samples were analyzed using a GENESYS 6 UV-vis Spectrophotometer (Thermo Scientific, Madison, WI, US) at a wavelength of $247 \mathrm{~nm}$. The standard curve was linear over the range of $1-50 \mu \mathrm{g} / \mathrm{mL}$ with an $R^{2}$ equal to 0.9998 .

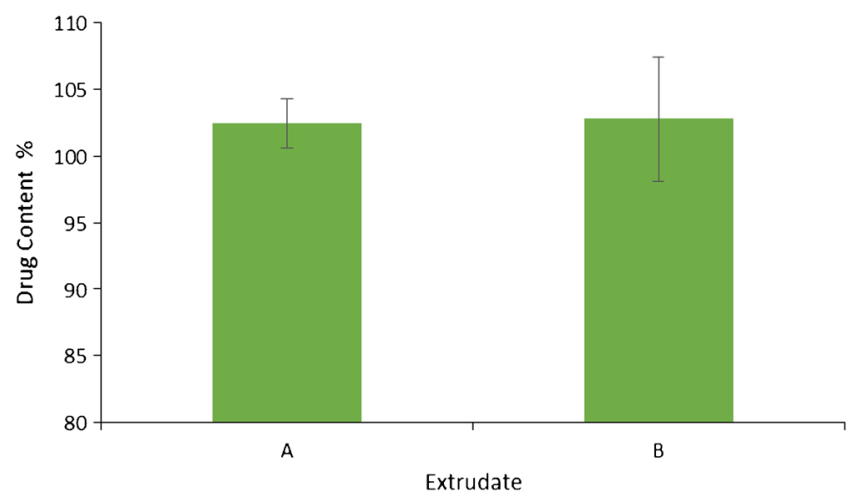

Fig. 6. Drug content for nanocrystal solid dispersion extrudate A and B 
Table II. LOD for NCSD and Soluplus ${ }^{\circledR}$ Extrudates

\begin{tabular}{ll}
\hline Extrudate & LOD (\%) \\
\hline Soluplus $®$ & $2.10 \pm 0.16$ \\
A & $2.24 \pm 0.07$ \\
B & $2.26 \pm 0.12$ \\
\hline
\end{tabular}

$L O D$ loss on drying

\section{Stability Test}

Stability studies were conducted to determine the effect of storage on the physical and chemical properties of the drug in two formulations (see Table I for formulation compositions). Milled extrudates were stored in screw-capped glass vials at realtime storage conditions $\left(25^{\circ} \mathrm{C} / 60\right.$ relative humidity $\left.[\mathrm{RH}]\right)$. Samples were taken at 1-, 2-, 3-, and 6-month intervals and characterized by dissolution and micromeritics studies. For the micromeritics studies, the extrudates were dispersed in ultrapurified water and then vortexed for $30 \mathrm{~s}$. The resulting dispersions were then analyzed using a Zetasizer Nano ZS (Malvern Instruments, Worcestershire, UK).

\section{RESULTS AND DISCUSSION}

\section{Preparation of EFZ NS}

Three different drug loadings $(2,4$, and $8 \% \mathrm{w} / \mathrm{v})$ were assessed to determine the highest drug content that can be used for NS. Before the HPH process, a regular homogenizing process (using a ULTRA-TURRAX ${ }^{\circledR}$ basic homogenizer) was carried out to pretreat the EFZ dispersion. This method significantly reduced the processing time for $\mathrm{HPH}$ as the size reduction was partially completed. The two formulations did not block HPH during processing. However, HPH blockage was observed during the first few cycles of $4 \%$ formulation processing. This issue was easily fixed by pretreating the formulation using a regular homogenizer. However, this was not true in the case of the $8 \%$ formulation as it blocked the HPH during processing, even after pretreatment, indicating that $8 \%$ drug loading was too high for the $\mathrm{HPH}$ used in this study. Thus, the highest drug loading that was used was $4 \%$.

Different cycles of homogenization were carried out to determine the optimum homogenization parameters (Fig. 1).
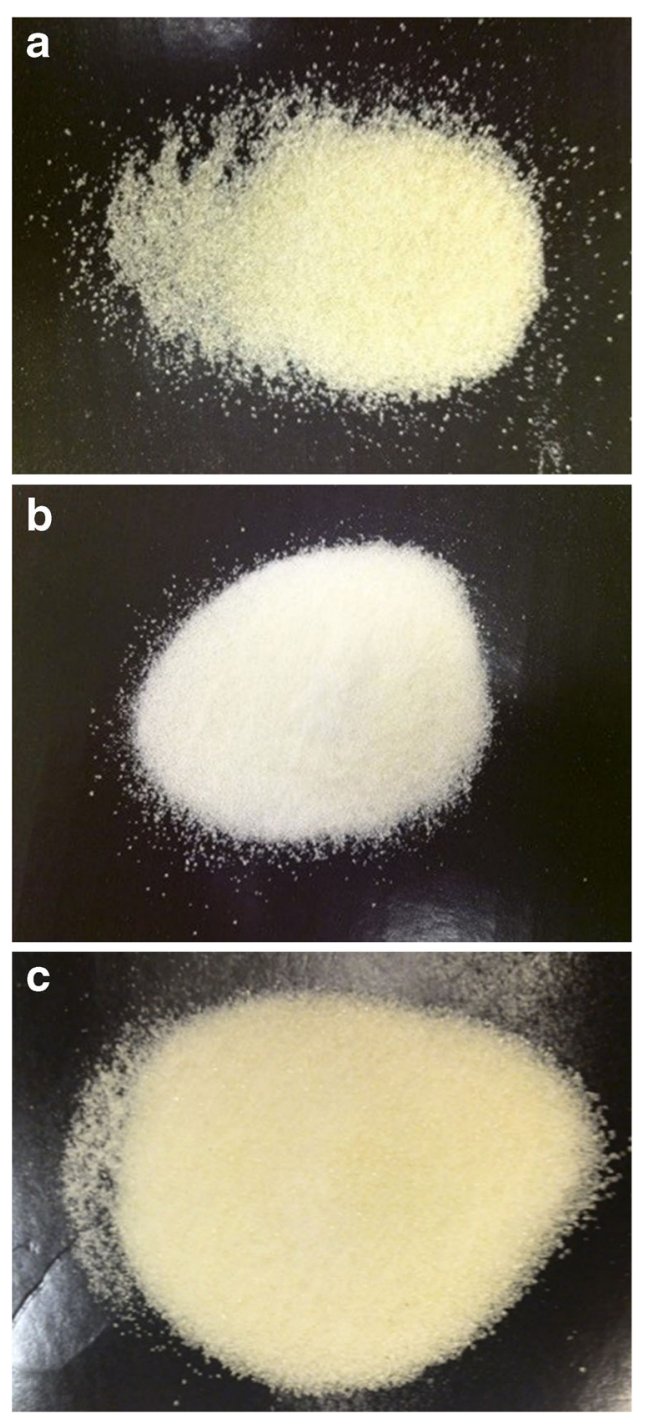

Fig. 8. Appearance of milled a extrudate $\mathrm{A}, \mathbf{b}$ extrudate $\mathrm{B}$, and $\mathbf{c}$ Soluplus ${ }^{\circledR}$

The particle size of NS A (2\% drug loading) was stable at around $320 \mathrm{~nm}$ after 20 homogenization cycles. Even after a few more homogenization cycles, there was no significant decrease in the particle size. Apart from particle size, polydispersity index (PDI) is also a very important parameter for NSs. A small PDI value indicates a narrow size distribution,

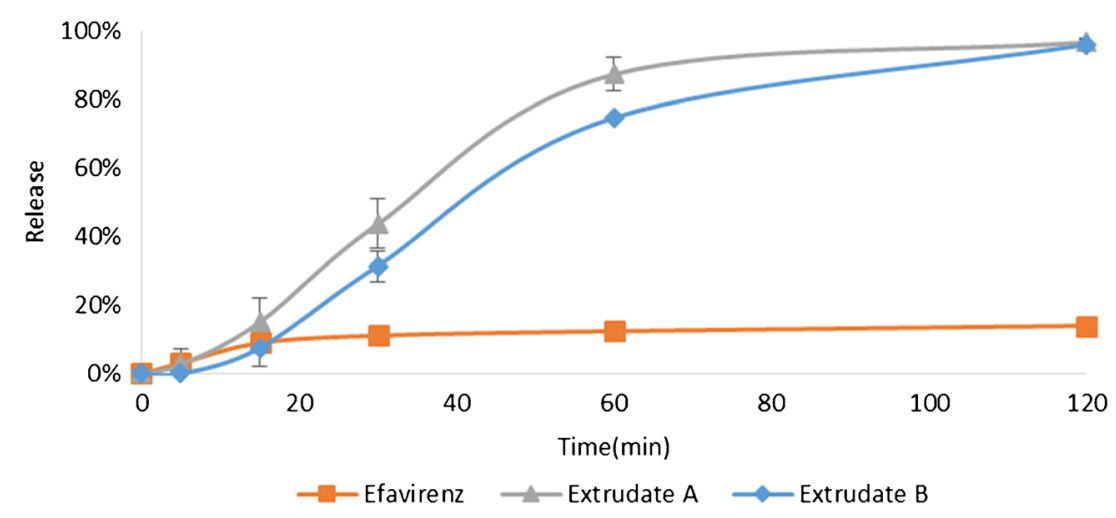

Fig. 7. In vitro drug release profiles for pure efavirenz, extrudate A, and extrudate $B$ 

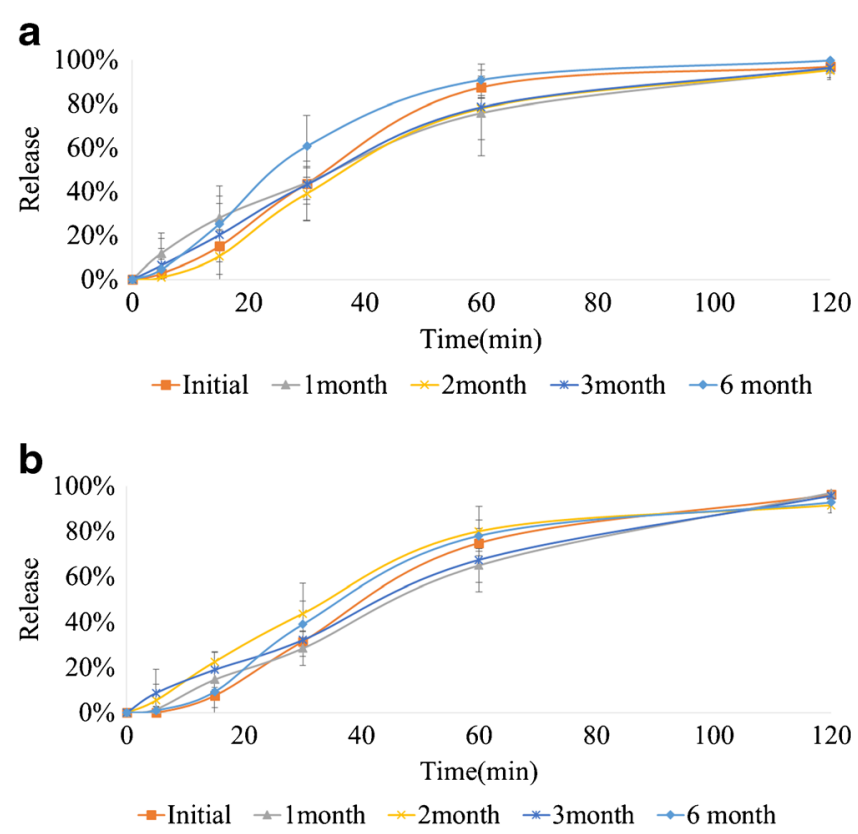

Fig. 9. In vitro release profiles of a extrudate $\mathrm{A}$ and $\mathbf{b}$ extrudate $\mathrm{B}$ stored at $25^{\circ} \mathrm{C} / 60 \%$ relative humidity

whereas a high PDI value indicates a broad size distribution. Usually, a PDI value below 0.5 is acceptable (34). The HPH process was optimized, and after 25 homogenization cycles for NS A, the PDI value decreased to below 0.5. Similarly, the $\mathrm{HPH}$ process was optimized for NS B (4\% drug loading), and after 45 homogenization cycles, the particle size was stable at around $350 \mathrm{~nm}$ and the PDI value was below 0.5 . It has been previously reported that a zeta potential of $\pm 20 \mathrm{mV}$ is sufficient to make a dispersion physically stable (35). In this study, the zeta potentials for all resulting homogeneous materials were in the range of -20 to $-30 \mathrm{mV}$, indicating that Kollidon ${ }^{\circledR}$ 30 and SLS were able to maintain the zeta potential within the desired range.

\section{Selection of Suitable Polymers}

The first step of the HME process was to choose an appropriate polymer. It is critical that the selected polymer is stable at the extrusion temperature and has the appropriate thermoplastic behavior to make melt extrusion possible (extrudable) (36). The glass transition temperature $\left(T_{\mathrm{g}}\right)$ is an important parameter affecting the extrudability of polymers because a high $T_{\mathrm{g}}$ will require a high temperature for processing, and in such situations, the API may degrade (37). As per the literature, the suitable $T_{\mathrm{g}}$ range for the $\mathrm{HME}$ process is considered to be between 50 and $180^{\circ} \mathrm{C}$ (38).

However, in this study, the $T_{\mathrm{g}}$ requirement is even more stringent. EFZ is a poorly water-soluble $(9.2 \mu \mathrm{g} / \mathrm{mL}, \mathrm{pH} 8.7$, $\left.25^{\circ} \mathrm{C}\right)$ lipophilic $(\log P=5.4)$ drug with a crystalline state. The melting temperature $\left(T_{\mathrm{m}}\right)$ of EFZ is around $140^{\circ} \mathrm{C}(39)$. To maintain the crystalline state of EFZ, the HME operating temperature must be kept below $140^{\circ} \mathrm{C}$. Generally, the extrusion process is carried out at least $20-40^{\circ} \mathrm{C}$ above the $T_{\mathrm{g}}(40)$; thus, there is a need to choose a polymer with a $T_{\mathrm{g}}$ below $100^{\circ} \mathrm{C}$. It is also important that the polymer is soluble in water in order to produce a homogeneous NCSD. Finally, there is a period when only the polymer is present in the barrel, so the operation temperature for the pure polymer is another important parameter.

Only a few polymers satisfy the above mentioned requirements, including Soluplus $®$, a graft copolymer composed of polyvinyl caprolactam-polyvinyl acetate-polyethylene glycol. It is a polymeric solubilizer with an amphiphilic chemical structure, making it an excellent solubilizer for poorly watersoluble drugs in aqueous media. Furthermore, it has a low $T_{\mathrm{g}}$ of about $70^{\circ} \mathrm{C}$, which also makes it a suitable choice for this study (41). The approximate temperature range for pure Soluplus ${ }^{\circledR}$ for use in HME was reported to be in the range of $120-200^{\circ} \mathrm{C}(42)$.

\section{Experimental Setup}

A schematic illustration of NCSD production by $\mathrm{HPH}-$ HME technique is shown in Fig. 2. Two parts were included in this system - a high-pressure homogenizer and a hot-melt

Table III. $f 2$ Values of Extrudates with Different Storage Times

\begin{tabular}{lcccc}
\hline & \multicolumn{2}{l}{$f_{2}$} & & \\
\cline { 2 - 5 } & 1 month & 2 months & 3 months & 6 months \\
\hline Extrudate A & 54.5 & 65.5 & 66.7 & 53.8 \\
Extrudate B & 63.9 & 52.9 & 58.7 & 70.7 \\
\hline
\end{tabular}


Table IV. Comparison of Particle Size, PDI, and Zeta Potential with Different Storage Times

\begin{tabular}{llll}
\hline Extrudate A & Particle size $(\mathrm{nm})$ & PDI & Zeta potential $(\mathrm{mV})$ \\
Initial & $28.04 \pm 1.35$ & $0.184 \pm 0.03$ & $-16.82 \pm 1.21$ \\
1 month & $28.04 \pm 0.17$ & $0.183 \pm 0.03$ & $-17.86 \pm 1.73$ \\
2 months & $29.78 \pm 6.81$ & $0.190 \pm 0.04$ & $-17.13 \pm 1.32$ \\
3 months & $29.60 \pm 4.33$ & $0.179 \pm 0.02$ & $-17.26 \pm 1.51$ \\
6 months & $29.05 \pm 0.26$ & $0.186 \pm 0.01$ & $-18.13 \pm 5.92$ \\
Extrudate B & Particle size $(\mathrm{nm})$ & PDI & Zeta potential (mV) \\
Initial & $34.11 \pm 5.03$ & $0.193 \pm 0.04$ & $14.38 \pm 2.10$ \\
1 month & $30.82 \pm 2.78$ & $0.189 \pm 0.02$ & $15.07 \pm 1.68$ \\
2 months & $30.67 \pm 2.93$ & $0.186 \pm 0.03$ & $14.68 \pm 1.52$ \\
3 months & $39.66 \pm 5.75$ & $0.185 \pm 0.03$ & $13.75 \pm 1.87$ \\
6 months & $32.94 \pm 0.88$ & $0.189 \pm 0.01$ & $13.93 \pm 0.76$ \\
\hline
\end{tabular}

$P D I$ polydispersity index

extruder. For this study, we used a modified screw configuration, as shown in Fig. 3.

The first zone of the extruder was used for feeding the polymer (Soluplus ${ }^{\circledR}$ ) into the extruder barrel using a feeding hopper. The elements used in this section were 2.0 L/D feed screws (conveying elements), which had sufficient free volume to take in the purging material for cleaning after processing. The $1.0 \mathrm{~L} / \mathrm{D}$ feed screws in zone 2 were used to compact the polymer and transport it forward. The temperature of $140^{\circ} \mathrm{C}$ applied to this section assisted in the melting of the polymer, thus decreasing the torque in the next mixing zone. The temperature of zone 3 was set at the same value as that of zone 2 . Three kinds of mixing elements with $30^{\circ}, 60^{\circ}$, and $90^{\circ}$ offset angles were successively assembled and installed in the barrel. This setting provided enough energy for the melting of the polymer and avoided any rapid increase in the torque. Zones 4 and 5 were both formed of conveying elements, and the NS can be injected in any one of these zones. The temperatures were set at 120 and $110^{\circ} \mathrm{C}$ for zones 4 and 5 , respectively, to avoid transformation of EFZ from the crystalline to the amorphous state. The water of the NS was expected to evaporate rapidly when the NS came into contact with the hot barrel and the molten polymer, and the EFZ nanocrystals would remain on the molten polymer. However, instant and complete evaporation of water was difficult to achieve, and a backflow phenomenon was observed after NS injection. Therefore, zone 5 was selected for the injection of the NS in order to provide more time and space for contact with the molten polymer, and to reduce the backflow of the NS. The $90^{\circ}$ mixing elements in zone 2 also assisted in this function. This screw configuration forced unevaporated NS to move back and forth between zone 4 and zone 5 until complete evaporation of water was achieved. Furthermore, this process would increase the contact between NS and the polymer, thus providing better distribution. Mixing elements with offset angles of $30^{\circ}$ and $60^{\circ}$ were used in zone 6 . The kneading of these elements contributed to the homogeneous distribution of the matrix material. A $90^{\circ}$ offset angle was not used in these mixing elements in order to avoid the generation and use of too much mechanical energy with the EFZ nanocrystals, which may result in the transformation of the crystalline state. The temperature for zone 6 and the remaining subsequent zones was set at $130^{\circ} \mathrm{C}$, which would further eliminate the remaining water in the matrix material. In zones 7 and 8 , 1.0 L/D feed screws were used to convey the matrix material to the die. Solidified extrudates coming out of the die were milled and processed for further analysis.

\section{Crystallinity and Morphology of Extrudate}

Differential scanning calorimetry was used to confirm the crystallinity of EFZ after extrusion (Fig. 4). A single sharp endotherm peak was observed for pure EFZ at about $140^{\circ} \mathrm{C}$ (Fig. 4a), which was identical to that of EFZ form I (39). Since the drug loading in the extrudate was low, the thermograph was magnified, as shown in Fig. 4b. The endotherm peak around $140^{\circ} \mathrm{C}$ reappeared in both extrudates A and B. This indicated that the EFZ maintained crystallinity after the extrusion.

SEM was used to determine the morphology and distribution of the EFZ particles (Fig. 5). Pure EFZ exists as sharp and long needles (Fig. 5a). The particle size of EFZ was around $20 \mu \mathrm{m}$. After HPH and HME, the particle size of EFZ was further decreased to less than $1000 \mathrm{~nm}$. The distribution of EFZ nanocrystals on Soluplus ${ }^{\circledR}$ is quite uniform (Fig. 5b). The distance between different EFZ nanocrystals prevents them from coming into contact with each other, and the size consistency will prevent the occurrence of Oswald ripening (43). These features thus establish the conjugated HPH-HME technique as a novel process to decrease the size of EFZ crystals that also aids in the uniform distribution of EFZ nanocrystals.

\section{Drug Content and Residual Moisture}

After extrusion, both extrudates were analyzed for drug content. The drug loadings of extrudates $\mathrm{A}$ and $\mathrm{B}$ were around 1.02 and $2.06 \%$, respectively. The drug contents of extrucates $\mathrm{A}$ and $\mathrm{B}$ were $102.47 \pm 1.87$ and $102.78 \pm 4.69 \%$, respectively (Fig. 6). This indicated that all the EFZ in the NS was enveloped in Soluplus ${ }^{\circledR}$. Considering the low drug loading in the extrudates, EFZ nanocrystals were regarded as homogeneously distributed in the extrudates.

The mean LOD values for the Soluplus ${ }^{\circledR}$ extrudate A, and extrudate B were $2.10,2.24$, and 2.26 , respectively (Table II), which indicates that there were no significant differences in residual moisture between these three samples ( $p$ value $>0.05$ ). Thus, it can be concluded that all the water that was added during the process was evaporated. 


\section{In Vitro Drug Release}

In vitro drug release was assessed for pure EFZ and both the extrudates (Fig. 7). As the aqueous solubility of EFZ is very low (recorded as $5.26 \pm 2.3 \mu \mathrm{g} / \mathrm{mL}$ ), $0.2 \%$ SLS was added to the dissolution media in order to increase the saturation solubility of EFZ, as well as to maintain the sink condition.

Owing to the poor solubility and wettability, only $14.04 \pm$ $0.80 \%$ of pure EFZ was released after 120 min of dissolution. Both extrudate $\mathrm{A}$ and $\mathrm{B}$ showed markedly enhanced dissolution rates with $96.86 \pm 0.99$ and $96.20 \pm 1.26 \%$ drug release overserved for extrudate A and B, respectively. It is evident that HPH-HME successfully increased the dissolution rate. This effect could have partially resulted from decreasing the particle size, or from the increase in the wettability of EFZ caused by Soluplus ${ }^{\circledR}$.

Although the same percentage drug release was reached at $120 \mathrm{~min}$, it was observed that the release from extrudate B was slower than that from extrudate A. The difference in the dissolution velocity may be because of the different ratios of Soluplus ${ }^{\circledR}$ and EFZ. In extrudate B, EFZ was surrounded by a relatively smaller amount of Soluplus ${ }^{\circledR}$ compared to that in extrudate A. The different appearances of milled extrudates $A$ and B supported this assumption (Fig. 8). In extrudate A, EFZ nanocrystals were completely covered by Soluplus ${ }^{\circledR}$. Therefore, the milled extrudate A was the same yellowish color as the pure Soluplus ${ }^{\circledR}$. However, the appearance of extrudate $\mathrm{B}$ was white, which may be because of the uncovered EFZ. This difference will result in slower wetting of EFZ, thus decreasing the dissolution velocity of extrudate $\mathrm{B}$.

However, the differences between these two formulations were not significant. The similarity factor $\left(f_{2}\right)$ was calculated to determine the similarity between the two formulations. The equation used to calculate $f_{2}$ is

$f_{2}=50 \times \log \left\{\left[1+(1 / n) \sum_{t=1}^{n}\left(R_{\mathrm{t}}-T_{\mathrm{t}}\right)^{2}\right]^{-0.5} \times 100\right\}$

where $R_{\mathrm{t}}$ and $T_{\mathrm{t}}$ are the dissolution value at time point $t$ of the reference and test product, respectively. According to US FDA guidance for industry, two dissolution profiles are considered similar when the $f_{2}$ value is greater than 50 (44). The calculated $f_{2}$ value was 55.0 , which indicated that the two formulations were similar.

\section{Stability Testing}

Stability testing was conducted for both formulations by storing at $25^{\circ} \mathrm{C} / 60 \% \mathrm{RH}$ for a period of 6 months. At various time points, samples were removed from storage and tested for in vitro drug release and micromeritics.

The release profiles for both the extrudates (A and B) were unchanged over the storage period of 6 months (Fig. 9). The $f_{2}$ values were calculated versus the initial extrudates for both the extrudates (Table III). All the $f_{2}$ values were greater than 50, which indicated that the release profiles obtained for both the extrudates at each time point were similar to the initial release profiles.

Comparison of particle size, PDI, and zeta potential was conducted for the extrudates at each time point (Table IV).
There was no significant change observed at any time point ( $p$ value $>0.05$ ).

All the above data indicates that the EFZ NCSD prepared by HPH-HME technique possesses good stability for a period of 6 months at $25^{\circ} \mathrm{C} / 60 \mathrm{RH}$.

\section{CONCLUSION}

The current work investigated the use of a novel conjugation of HPH and HME techniques to continuously produce NCSD. This technique helps to overcome the nanocrystal formulation problems that are associated with the conventional methods. By using this technique, an increase in the dissolution rate was achieved as a result of the decreased particle size and increased surface area, and also owing to the improved wettability. The good stability was attributed to the maintained crystalline state of the drug.

\section{ACKNOWLEDGMENTS}

This project was partially supported by Grant Number P20GM104932 from the National Institute of General Medical Sciences (NIGMS), a component of NIH. The authors would also like to thank Dr. Vijayasankar Raman of the National Center for Natural Products Research, School of Pharmacy, The University of Mississippi, for his valuable assistance with the SEM imaging studies.

\section{REFERENCES}

1. Speiser P. Poorly soluble drugs: a challenge in drug delivery. In: Muller RH, Benita S, Bohm B, editors. Emulsions and nanosuspensions for the formulation of poorly soluble drugs. Stuttgart: Medpharm Scientific Publishers; 1998. p. 15-28.

2. Savjani KT, Gajjar AK, Savjani JK. Drug solubility: importance and enhancement techniques. ISRN Pharm. 2012;2012:195727.

3. Gribbon P, Andreas S. High-throughput drug discovery: what can we expect from HTS? Drug Discov Today. 2005;10(1):17-22.

4. Leuner C, Dressman J. Improving drug solubility for oral delivery using solid dispersions. Eur J Pharm Biopharm. 2000;50(1):47-60.

5. Vasconcelos T, Sarmento B, Costa P. Solid dispersions as strategy to improve oral bioavailability of poor water soluble drugs. Drug Discov Today. 2007;12(23-24):1068-75.

6. Sweetana S, Akers MJ. Solubility principles and practices for parenteral drug dosage form development. PDA J Pharm Sci Technol/PDA. 1995;50(5):330-42.

7. Szejtli J. Cyclodextrin technology. Netherlands: Springer Science \& Business Media; 1988.

8. Chiou WL, Riegelman S. Pharmaceutical applications of solid dispersion systems. J Pharm Sci. 1971;60(9):1281-302.

9. Alshahrani SM, Lu W, Park JB, Morott JT, Alsulays BB, Majumdar S, et al. Stability-enhanced hot-melt extruded amorphous solid dispersions via combinations of soluplus(R) and HPMCAS-HF. AAPS PharmSciTech. 2015;16(4):824-34.

10. Feng X, Ye X, Park JB, Lu W, Morott J, Beissner B, et al. Evaluation of the recrystallization kinetics of hot-melt extruded polymeric solid dispersions using an improved Avrami equation. Drug Dev Ind Pharm. 2014. doi:10.3109/03639045.2014.958755.

11. Yang J, Grey K, Doney J. An improved kinetics approach to describe the physical stability of amorphous solid dispersions. Int J Pharm. 2010;384(1):24-31.

12. Junghanns J-UA, Müller RH. Nanocrystal technology, drug delivery and clinical applications. Int J Nanomedicine. 2008;3(3):295. 
13. Noyes AA, Whitney WR. The rate of solution of solid substances in their own solutions. J Am Chem Soc. 1897;19(12):930-4.

14. Müller RH, Benita S, Bohm B. Emulsions and nanosuspensions for the formulation of poorly soluble drugs. Stuttgart: medpharm GmbH Scientific Publishers; 1998.

15. Shegokar R, Muller RH. Nanocrystals: industrially feasible multifunctional formulation technology for poorly soluble actives. Int J Pharm. 2010;399(1-2):129-39.

16. Van Eerdenbrugh B, Van den Mooter G, Augustijns P. Top-down production of drug nanocrystals: nanosuspension stabilization, miniaturization and transformation into solid products. Int $\mathbf{J}$ Pharm. 2008;364(1):64-75.

17. Sinha B, Muller RH, Moschwitzer JP. Bottom-up approaches for preparing drug nanocrystals: formulations and factors affecting particle size. Int J Pharm. 2013;453(1):126-41.

18. Moschwitzer JP. Drug nanocrystals in the commercial pharmaceutical development process. Int J Pharm. 2013;453(1):142-56.

19. Muller RH, Moschwitzer JP, Bushrab FN. Manufacturing of nanoparticles by milling and homogenization techniques. In: Gupta RB, Kompella UB, editors. Nanopartical technology for drug delivery. New York: Taylor \& Francis; 2006. p. 21-52.

20. Shah S, Maddineni S, Lu J, Repka MA. Melt extrusion with poorly soluble drugs. Int J Pharm. 2013;453(1):233-52.

21. Crowley MM, Zhang F, Repka MA, Thumma S, Upadhye SB, Kumar Battu S, et al. Pharmaceutical applications of hot-melt extrusion: part I. Drug Dev Ind Pharm. 2007;33(9):909-26.

22. Patil H, Tiwari RV, Repka MA. Hot-melt extrusion: from theory to application in pharmaceutical formulation. AAPS PharmSciTech. 2015. doi:10.1208/s12249-015-0360-7.

23. Follonier N, Doelker E, Cole ET. Evaluation of hot-melt extrusion as a new technique for the production of polymer-based pellets for sustained release capsules containing high loadings of freely soluble drugs. Drug Dev Ind Pharm. 1994;20(8):1323-39.

24. Crowley MM, Schroeder B, Fredersdorf A, Obara S, Talarico M, Kucera S, et al. Physicochemical properties and mechanism of drug release from ethyl cellulose matrix tablets prepared by direct compression and hot-melt extrusion. Int $\mathrm{J}$ Pharm. 2004;269(2):509-22

25. Aitken-Nichol C, Zhang F, McGinity JW. Hot melt extrusion of acrylic films. Pharm Res. 1996;13(5):804-8.

26. Chen M, Lu J, Deng W, Singh A, Mohammed NN, Repka MA, et al. Influence of processing parameters and formulation factors on the bioadhesive, temperature stability and drug release properties of hot-melt extruded films containing miconazole. AAPS PharmSciTech. 2014;15(3):522-9.

27. Rothen-Weinhold A, Oudry N, Schwach-Abdellaoui K, FrutigerHughes S, Hughes GJ, Jeannerat D, et al. Formation of peptide impurities in polyester matrices during implant manufacturing. Eur J Pharm Biopharm. 2000;49(3):253-7.

28. Patil H, Kulkarni V, Majumdar S, Repka MA. Continuous manufacturing of solid lipid nanoparticles by hot melt extrusion. Int J Pharm. 2014;471(1):153-6.

29. Patil H, Feng X, Ye X, Majumdar S, Repka MA. Continuous production of fenofibrate solid lipid nanoparticles by hot-melt extrusion technology: a systematic study based on a quality by design approach. AAPS J. 2015;17(1):194-205.
30. Baumgartner R, Eitzlmayr A, Matsko N, Tetyczka C, Khinast J, Roblegg E. Nano-extrusion: a promising tool for continuous manufacturing of solid nano-formulations. Int J Pharm. 2014;477(1-2):1-11.

31. Patel GV, Patel VB, Pathak A, Rajput SJ. Nanosuspension of efavirenz for improved oral bioavailability: formulation optimization, in vitro, in situ and in vivo evaluation. Drug Dev Ind Pharm. 2013;40(1):80-91.

32. Wang Y, Zheng Y, Zhang L, Wang Q, Zhang D. Stability of nanosuspensions in drug delivery. J Control Release. 2013;172(3):1126-41.

33. Singh A, Majumdar S, Deng W, Mohammed N, Chittiboyina A, Raman V, et al. Development and characterization of taste masked Efavirenz pellets utilizing hot melt extrusion. J Drug Delivery Sci Technol. 2013;23(2):157-63.

34. Mohammadi Z, Abolhassani M, Dorkoosh F, Hosseinkhani S, Gilani K, Amini T, et al. Preparation and evaluation of chitosan-DNA-FAP-B nanoparticles as a novel non-viral vector for gene delivery to the lung epithelial cells. Int J Pharm. 2011:409(1):307-13.

35. Shah RM, Malherbe F, Eldridge D, Palombo EA, Harding IH. Physicochemical characterization of solid lipid nanoparticles (SLNs) prepared by a novel microemulsion technique. J Colloid Interface Sci. 2014;428:286-94.

36. PAGE NMA, PAGE C, PAGE C. Suitability of plasticized polymers for hot melt extrusion.PBP World Meeting. March 8-11, 2010. Valletta, Malta.

37. Meena A, Parikh T, Gupta SS, Serajuddin AT. Investigation of thermal and viscoelastic properties of polymers relevant to hot melt extrusion, II: cellulosic polymers. J Excip Food Chem. 2014;5(1):46-55.

38. Kolter K, Maschke A. Melt extrusion for pharmaceuticals. ExAct. 2009:22:2-5.

39. Chadha R, Arora P, Saini A, Jain DS. An insight into thermodynamic relationship between polymorphic forms of efavirenz. J Pharm Pharm Sci. 2012;15(2):234-51.

40. Karl M, Nalawade S, Maschke A, Djuric D, Kolter K, editors. Suitability of pure and plasticized polymers for hot melt extrusion. The 37th Annual Meeting and Exposition of the Controlled Release Society. Portland: Oregon Convention Center; 2010

41. Hardung H, Djuric D, Ali S. Combining HME \& solubilization: Soluplus ${ }^{\circledR}$ - the solid solution. Drug Deliv Technol. 2010;10(3):20-7.

42. Ali S, Langley N, Djuric D, Kolter K. Eye on excipients. http:// pharma-ingredients.basf.com/Documents/ENP/Articles/EN/ EyeOnExcipients_1010TC.pdf. Accessed 3 March 2015.

43. Ostwald W. Lehrbuch der allgemeinen Chemie. Leipzig: Engelmann; 1896.

44. FDA US. Guidance for Industry Waiver of In Vivo Bioavailability and Bioequivalence Studies for Immediate-Release Solid Oral Dosage Forms Based on a Biopharmaceutics Classification System. In: Center for Drug Evaluation and Research (CDER). 2000. http://www.fda.gov/downloads/Drugs/Guidances/ ucm070246.pdf. Accessed 3 March 2015. 